\title{
DESENVOLVIMENTO DE UM SISTEMA DE MEDIÇÃO, MONITORAMENTO E CONTROLE DO TAMANHO DE BOLHA EM UMA COLUNA DE FLOTAÇÃO MINIPILOTO
}

\author{
C. OLIVEIRA ${ }^{2}$, A. A. POMPEO ${ }^{1}$, M. A. SEGER ${ }^{1}$ e R. T. RODRIGUES ${ }^{*}$ \\ ${ }^{1}$ Universidade Federal do Rio Grande do Sul (UFRGS), Departamento de Engenharia de Minas \\ ${ }^{2}$ UniLaSalle, Mestrado em Avaliação em Impactos Ambientais em Mineração \\ rafael.teixeira@ufrgs.br*
}

Artigo submetido em novembro/2015 e aceito em novembro/2015

DOI: $10.15628 /$ holos.2015.3679

\section{RESUMO}

Apesar da grande importância atribuída ao tamanho das bolhas no processo de flotação, ainda não existem muitos equipamentos disponíveis para efetuar a análise deste parâmetro, principalmente, em estudos técnicocientíficos (em escala de bancada e piloto), essenciais para o desenvolvimento e otimização dos processos em escala industrial. Neste contexto, o objetivo do presente trabalho foi o desenvolvimento de um sistema online para medir, monitorar e controlar o tamanho de bolhas para estudos de flotação em coluna minipiloto. O sistema proposto, nas condições experimentais aplicadas, permitiu medir e controlar o diâmetro médio de bolha entre 1 e $3 \mathrm{~mm}$. O sistema também permitiu realizar medidas de holdup e estimativas do fluxo de área superficial de bolhas (Sb), com valores entre $5-12 \%$ e 10 $25 \mathrm{~m}^{2} / \mathrm{m}^{2} \mathrm{~s}$, respectivamente. Os resultados mostraram que o sistema desenvolvido representa um avanço nos estudos científicos sobre flotação, pois permite a determinação, monitoramento e controle do tamanho de bolhas durante a realização dos experimentos, melhorando o controle operacional do processo com maior conhecimento do efeito da distribuição do tamanho de bolha na flotação de diferentes minérios.

PALAVRAS-CHAVE: automação, controle, tamanho de bolha, flotação, análise de imagens.

\section{DEVELOPMENT OF A SYSTEM FOR BUBBLE SIZE MEASUREMENT, MONITORING AND CONTROL IN A BENCH SCALE COLUMN FLOTATION}

\begin{abstract}
Despite the great importance attributed to bubble size in the flotation process there are not yet many pieces of equipment available to perform the analysis of this parameter, mainly, in scientific-technique studies (batch and pilot scale) essential to the development and optimization of the processes in industrial scale. In this context, the aim of the present work was the development of an online system to measure, monitor and control the bubble size in flotation studies in a mini pilot column flotation. The proposed system and the applied experimental conditions allowed to measure and control the mean bubble diameter between 1 and $3 \mathrm{~mm}$.
\end{abstract}

The system also enabled measurements of holdup and estimates of the bubble surface area flux (Sb) simultaneously, with values between 5-12 \% and 12-25 $\mathrm{m}^{2} / \mathrm{m}^{2} \mathrm{~s}$, respectively. The results showed that the developed system represents an advance in the scientific studies about flotation, because it allows the determination, monitoring and control of the bubble size during the experimental runs, improving the operational control of the process with greater knowledge of the effect of bubble size distribution in flotation of different ores.

KEYWORDS: automation, process control, bubble size, flotation, image analysis 


\section{INTRODUÇÃO}

Diversos sistemas utilizados para determinação do tamanho de bolha têm sido reportados na literatura, dentre os principais destacam-se os que utilizam um método óptico para análise de bolhas succionadas por um tubo capilar (Gorain et al., 1995), bem como os métodos baseados na captura e análise de imagens digitais utilizando visores externos (Chen et al.; 2001; Grau e Heiskanen, 2002; Rodrigues, 2004; Hernandez-Aguilar et al., 2004; Oliveira et al., 2011; Pompeo et al., 2013). Dentre as técnicas propostas, os métodos que utilizam o conceito da visualização de bolhas em uma câmara externa, combinando técnicas de captura, processamento e análise de imagens digitais aparecem como soluções promissoras para medições online de tamanho de bolhas em células de flotação industriais. Neste contexto podemos destacar os equipamentos desenvolvidos pela McGill University (Hernandez-Aguilar et al., 2004), HUT/Helsinki University of Technology (Grau e Heiskanen, 2002) e o equipamento comercializado pela Stone Three (África do Sul) denominado de Anglo Platinum Bubble Sizer (www.stonethreemining.com).

Entretanto, estes equipamentos possuem aplicações em equipamentos industriais, visto que realizam uma amostragem de parte do fluxo de bolhas pela parte superior do equipamento de flotação, tipicamente através de um tubo amostrador que coleta (conduz) as bolhas desde o interior da polpa até a câmara de visualização externa. Deste modo, não podem ser aplicados em equipamentos de bancada ou piloto de pequeno porte, pois por serem invasivos, retiram parte do fluxo, além do fato de acumularem partículas sólidas no interior da câmara de visualização (fato inerente a amostragem do fluxo de agregados bolha-partículas), o que torna a obtenção de imagens por luz transmitida mais difícil a medida que o ensaio avança, sendo necessário paradas frequentes para limpeza da câmara de visualização.

Os parâmetros relacionados à dispersão ar-polpa no processo de flotação incluem, além de medidas de velocidade superficial de gás $(\mathrm{Jg})$ e do holdup de gás $\left(\varepsilon_{\mathrm{g}}\right)$, o tamanho de bolhas (normalmente o diâmetro médio de Sauter, $D_{32}$ ), o qual permite a estimativa do fluxo superficial de bolhas (Sb) (Finch et al., 2000), definido como: $\mathrm{Sb}=6 \mathrm{Jg} / \mathrm{D}_{32}$. O Sb tem sido amplamente utilizado para avaliar a eficiência de dispersão do gás em sistemas de flotação, assim como na estimativa da capacidade de carregamento de zonas de coleção em células de flotação. Desta forma, uma determinação mais precisa da distribuição de tamanho de bolha (DTB), e consequentemente do $D_{32}$, permitirá também uma estimativa mais precisa do Sb.

Apesar da grande importância atribuída ao tamanho das bolhas no processo de flotação, poucos são os equipamentos disponíveis para esta análise, principalmente no que se refere a equipamentos de flotação destinados a estudos técnico-científicos em pequena escala, como os equipamentos de bancada e piloto. $O$ objetivo deste trabalho foi o desenvolvimento de um sistema online de medição, monitoramento e controle do tamanho de bolha para ensaios de flotação em coluna minipiloto. O sistema representa um grande avanço para a realização de estudos científicos sobre a flotação, pois determina, monitora e controla o tamanho de bolha durante a realização de ensaios e deste modo, servirá para aumento do controle das condições operacionais dos ensaios de flotação, assim como maior entendimento e conhecimento do efeito da distribuição do tamanho de bolha no processo de flotação para diferentes minérios. 


\section{METODOLOGIA}

O equipamento proposto utiliza o princípio da captura, processamento e análise de imagens digitais, conforme descrito por Oliveira et al. (2011) e Pompeo et al. (2013), entretanto, foram realizadas modificações para permitir a medição e controle online do tamanho das bolhas geradas na base de colunas de flotação. A medição do tamanho das bolhas foi realizada previamente a entrada das mesmas na coluna de flotação. Deste modo, o fluxo de polpa não prejudica a visualização das bolhas, garantindo, desta forma, a qualidade das imagens e permitindo a operação de forma contínua por longos períodos de tempo, sendo estes os principais inconvenientes desta técnica segundo Grau e Heiskanen (2002).

A Figura 1 apresenta um desenho esquemático do sistema de medição, monitoramento e controle do tamanho de bolha em uma coluna de flotação minipiloto. O sistema era composto por duas partes principais: 1- Câmara de geração de bolhas e 2-Câmara de visualização para obtenção de imagens digitais. As bolhas geradas, de forma controlada, na câmara de geração de bolhas ascendem até a câmara de visualização de onde foram obtidas imagens digitais antes de ingressarem na base da coluna de flotação. A câmara de geração de bolhas consiste de uma câmara tubular-cônica destinada à geração de bolhas através de spargers tipicamente utilizados para este fim, como tubos porosos e constrições de fluxo (placa de orifício, venturi, entre outros). O controle automatizado do tamanho de bolha foi realizado variando-se a dosagem (vazão e /ou concentração) de tensoativo injetado na câmara de geração de bolhas, de forma manual ou automatizada tendo como referência o tamanho de bolha medido.

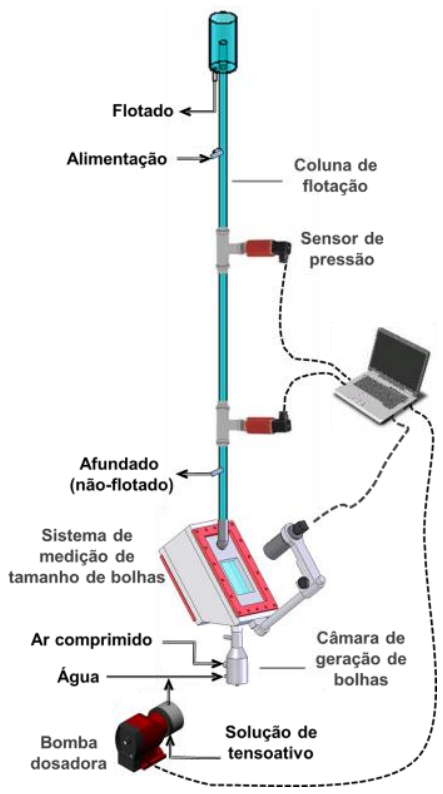

Figura 1. Montagem experimental para estudos de determinação de tamanho de bolha online em uma coluna de flotação minipiloto.

As imagens foram capturadas com uma câmera SONY XC-HR70 (1024 x 768 pixels), acoplada a uma lente de zoom (MLH-10X Macro Zoom - Moritex Corporation). As etapas de captura, processamento e análise das imagens foram automatizadas com o auxílio de um software de análise de 
imagens (Image Tool 3.0). O tempo para aquisição, processamento e análise de cada imagem foi de $10 \mathrm{~s}$. A iluminação do sistema foi realizada por uma placa de LED de $10 \times 10 \mathrm{~cm}$. A maior área fotografada (campo de visão) foi de $44,1 \mathrm{~cm}^{2}(7,67 \times 5,75 \mathrm{~cm})$, o que representa praticamente a totalidade da largura da janela de visualização. Foram realizados ensaios acoplando-se o equipamento de medição de tamanho de bolha em uma coluna de flotação minipiloto de $27,5 \mathrm{~mm}$ de diâmetro interno e $210 \mathrm{~cm}$ de altura, conforme configuração apresentada na Figura 1. Para geração de bolhas utilizou-se um tubo poroso, fabricado em pó de aço inox sinterizado, com medidas externas de 15 x $30 \mathrm{~mm}$ (diâmetro x comprimento) e espessura de parede de $2,3 \mathrm{~mm}$.

Os ensaios realizados foram conduzidos somente com água, nas condições operacionais descritas na Tabela 1.

Tabela 1. Condições operacionais dos ensaios para determinação do tamanho de bolha em uma coluna de flotação minipiloto.

\begin{tabular}{lc}
\hline \multicolumn{1}{c}{ Parâmetro } & Valor \\
\hline Vazão de alimentação da coluna & $70 \mathrm{~mL} / \mathrm{min}$ \\
Velocidade superficial de alimentação $\left(\mathrm{J}_{\mathrm{sl}}\right)$ & $0,20 \mathrm{~cm} / \mathrm{s}$ \\
Vazão de ar & $180 \mathrm{~mL} / \mathrm{min}$ \\
Velocidade superficial do gás $\left(\mathrm{J}_{\mathrm{g}}\right)$ & $0,52 \mathrm{~cm} / \mathrm{s}$ \\
Tipo de Espumante & Éter Metílico de Polipropilenoglicol (nome \\
Dosagens de Espumante & comercial: DF250) \\
\hline
\end{tabular}

A injeção de espumante DF250 iniciou 10 minutos após o início de cada ensaio. Todos os estudos foram realizados a temperatura ambiente $\left(\sim 25{ }^{\circ} \mathrm{C}\right)$, com água da rede municipal de abastecimento de Porto Alegre/RS (DMAE) e pH natural ( 7). Todos os ensaios foram realizados em duplicata ou triplicata. O holdup foi determinado através de medidas de pressão realizadas por dois sensores de pressão instalados na zona de recuperação da coluna de flotação. O holdup do ar (Eg) foi calculado pela seguinte equação:

$$
\varepsilon_{g}=1-\frac{\Delta P}{\rho_{S L} \cdot g \cdot L}
$$

onde: $\Delta \mathrm{P}=$ diferença de pressão entre os dois pontos da zona de recuperação $(\mathrm{kPa}) ; \rho_{\mathrm{SL}}=$ densidade da polpa entre esses pontos $\left(\mathrm{g} / \mathrm{cm}^{3}\right) ; \mathrm{L}=$ distância entre os pontos das medidas de pressão $(\mathrm{m}) ; \mathrm{g}=$ aceleração da gravidade $\left(\mathrm{m} / \mathrm{s}^{2}\right)$.

$\mathrm{Na}$ análise das imagens foi utilizado o diâmetro de Feret médio (medido em 8 ângulos) como sendo o diâmetro de bolha. $O$ diâmetro médio adotado foi o diâmetro médio de Sauter $\left(D_{32}\right)$, definido como o diâmetro médio volume-superfície, conforme a Equação 2.

$$
\mathrm{d}_{\text {sauter }}=\frac{\sum \mathrm{n}_{\mathrm{i}} \mathrm{d}_{\mathrm{bi}}^{3}}{\sum \mathrm{n}_{\mathrm{i}} \mathrm{d}_{\mathrm{bi}}^{2}}
$$


onde, os parâmetros $d_{b}$ e $n$ são o diâmetro de bolha e o número de bolhas para cada classe de tamanho " $i$ ", respectivamente.

\section{RESULTADOS E DISCUSSÕES}

Foi possível obter diferentes tamanhos de bolha, numa faixa entre 1 e $3 \mathrm{~mm}$, segundo as condições experimentais empregadas A Figura 2 mostra exemplos de imagens capturadas para diferentes concentrações de espumante (DF250).

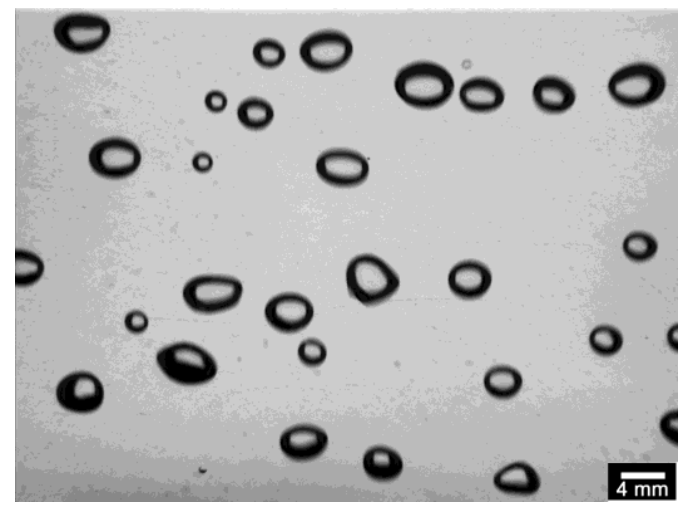

(A) sem DF250.

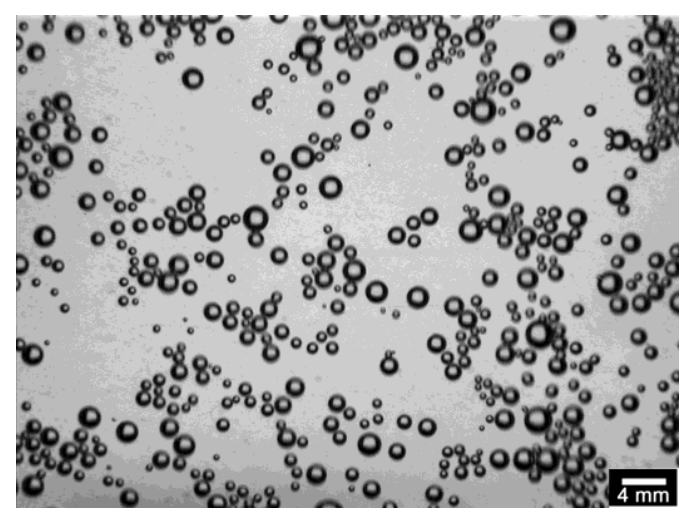

(C) $15,0 \mathrm{mg} / \mathrm{L} \mathrm{de} \mathrm{DF250}$

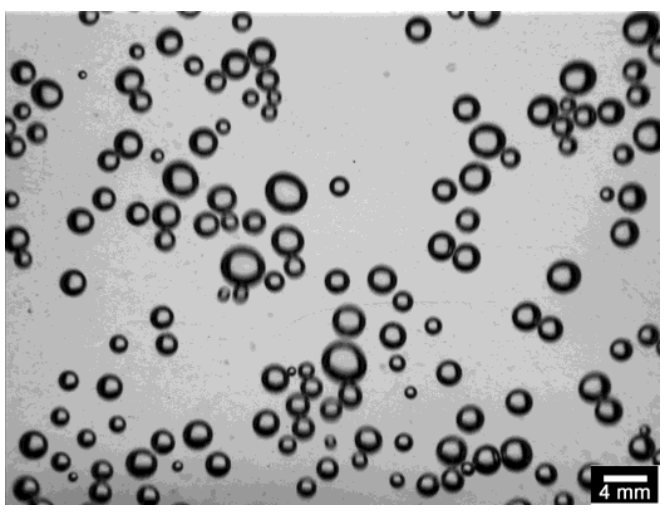

(B) 4,0 mg/L de DF250.

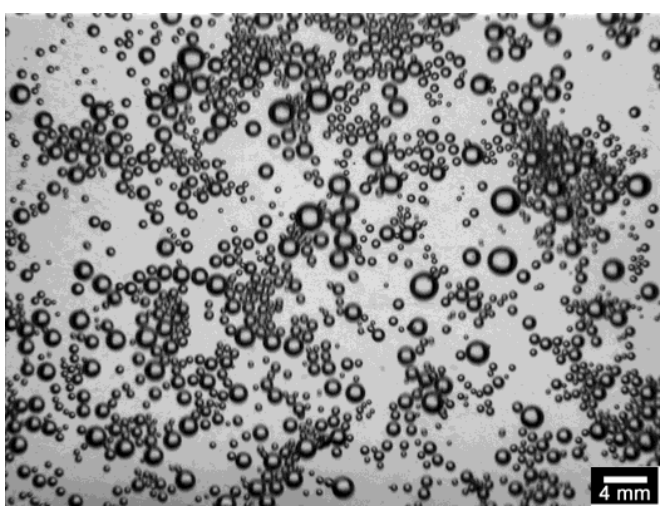

(D) $62 \mathrm{mg} / \mathrm{Lde}$ DF250

Figura 2. Típicas imagens capturadas para diferentes dosagens de tensoativo DF250.

A Figura 3 apresenta os tamanhos médios de bolha e de holdup em função das dosagens de espumante (DF250). O tamanho de bolha inicial, ou seja, antes do início da injeção de tensoativo, foi de aproximadamente $3,55 \mathrm{~mm}$ para todos os ensaios. Com a injeção contínua de DF250, o diâmetro médio atingiu valores constantes em poucos segundos $\left(D_{32}=2,58 \mathrm{~mm}\right.$ para $4 \mathrm{mg} / \mathrm{L}$ DF250; $D_{32}=1,69$ $\mathrm{mm}$ para $15 \mathrm{mg} / \mathrm{L} \mathrm{DF} 250$ e $\mathrm{D}_{32}=1,36 \mathrm{~mm}$ para $62 \mathrm{mg} / \mathrm{L}$ de DF 250). Como esperado, o comportamento do holdup foi mais lento e levou, em média, 30 minutos para atingir valores constantes. Esta resposta mais lenta do holdup deve-se a coalescência das bolhas na câmara de visualização, assim como na coluna de flotação. Entretanto, este inconveniente pode ser minimizado se a concentração de tensoativo na câmara de geração de bolhas estiver acima da concentração crítica de coalescência (CCC), conforme reportado por Cho e Laskowski (2002). Segundo Cho e Laskowski (2002) a dosagem de espumante é importante para avaliação de outros parâmetros operacionais e geometria do sparger, pois trabalhando-se em concentrações acima da CCC, ou seja, minimizando a coalescência, pode-se avaliar o real efeito de outros parâmetros. 


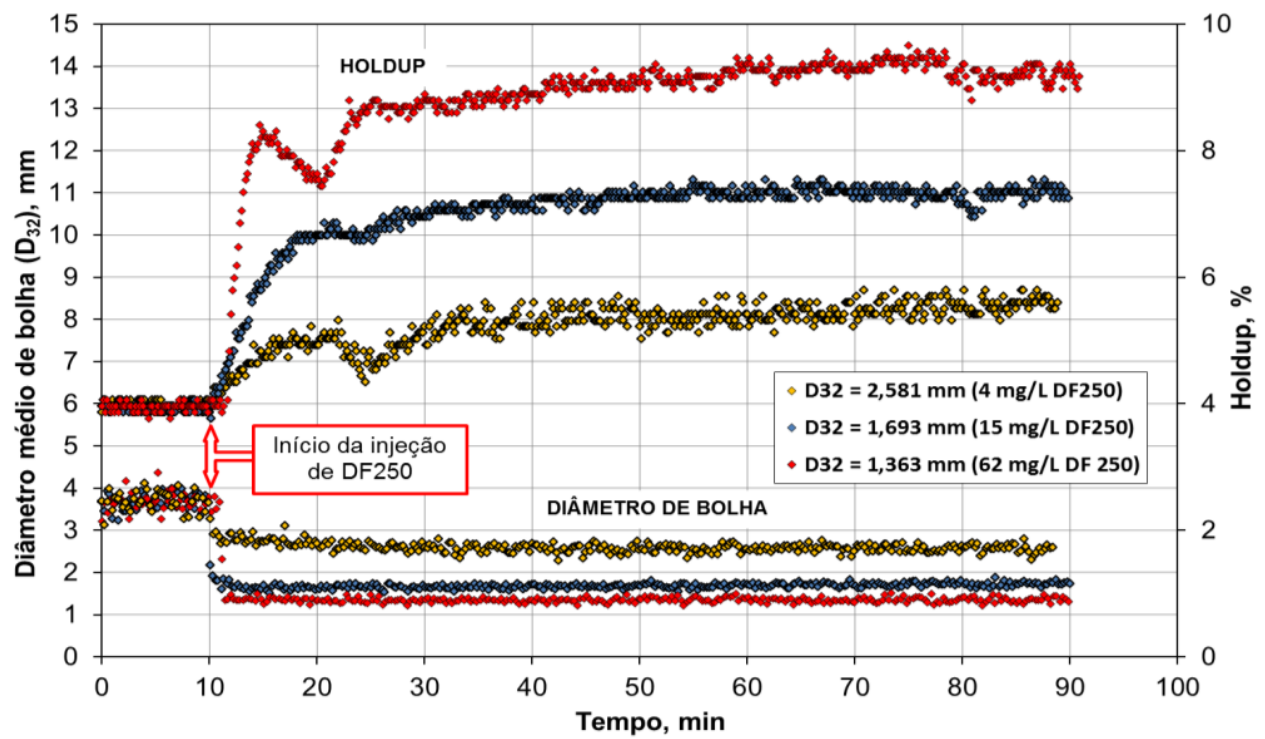

Figura 3. Tamanho de bolha $\left(D_{32}\right)$ e holdup em função do tempo, para diferentes dosagens de tensoativo DF250.

As Figuras 4 e 5 mostram o efeito do diâmetro médio de bolha no holdup $\left(\varepsilon_{\mathrm{g}}\right)$ e no bubble surface área flux (Sb), os quais apresentaram a seguinte relação: $\mathrm{Sb} \cong 2,51 \varepsilon_{\mathrm{g}}$. Conforme reportado por Finch e Dobby (1990), o holdup apresenta um crescimento exponencial com relação ao tamanho de bolha. Segundo Finch et al. (2000), holdup e Sb apresentam uma relação linear ( $S b \cong 5,5 \varepsilon_{\mathrm{g}}$ ). Entretanto, Grau e Heiskanen (2003) também encontraram valores bem menores de Sb (27 - 36 $\mathrm{m}^{2} / \mathrm{m}^{2} \mathrm{~s}$ ) em relação aos valores comumente reportados, na faixa $36-200 \mathrm{~m}^{2} / \mathrm{m}^{2}$ (Gorain et al., 1997). Grau e Heiskanen (2003) atribuem a estes valores elevados de Sb uma subestimação do diâmetro de bolha.

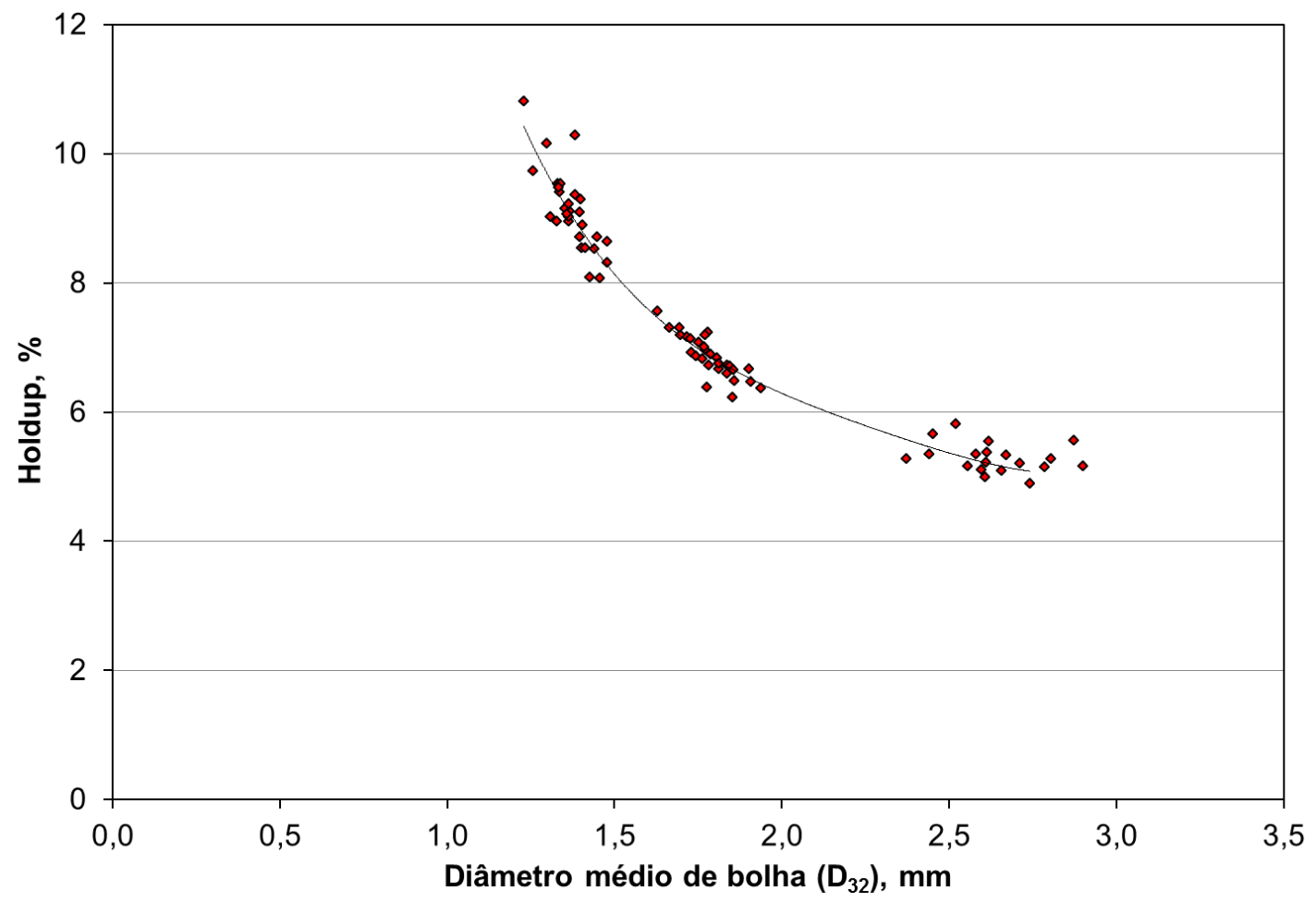

Figura 4. Relação entre o diâmetro médio de bolha e o holdup. Condições experimentais: $Q_{a r}=180 \mathrm{ml} / \mathrm{min} ; \mathrm{J}_{\mathrm{g}}=0,52$ $\mathrm{cm} / \mathrm{s}, Q_{\text {alimentação }}=70 \mathrm{ml} / \mathrm{min} ; J_{\mathrm{sl}}=0,20 \mathrm{~cm} / \mathrm{s} ;[D F 250]=4-62 \mathrm{mg} / \mathrm{L}$. 
Entre as vantagens inerentes a medição em tempo real (online) do tamanho de bolhas destacam-se: maior consistência na operação e nos resultados metalúrgicos; histórico de dados de tamanho de bolha; alta frequência de medição; medidas precisas tornam possível o controle automatizado do tamanho de bolha; possibilidade de cálculo de outros parâmetros, como por exemplo, o fluxo superficial de bolhas (Sb), maior reprodutibilidade dos resultados.

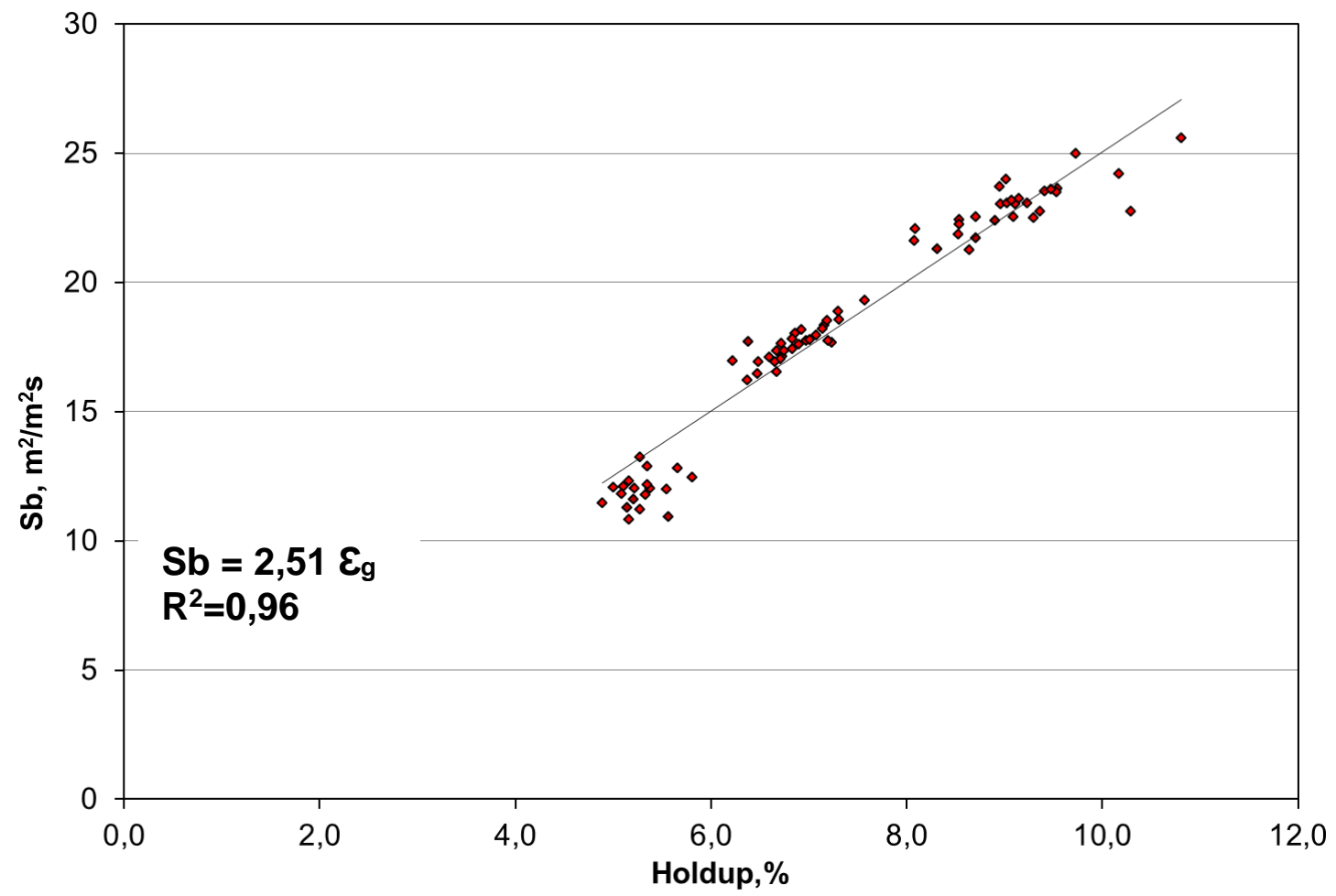

Figura 5. Relação entre o holdup e o fluxo superficial de bolhas (Sb). Condições experimentais: $Q_{a r}=180 \mathrm{ml} / \mathrm{min} ; J_{\mathrm{g}}=$ $0,52 \mathrm{~cm} / \mathrm{s}, Q_{\text {alimentaç̃o }}=70 \mathrm{ml} / \mathrm{min} ; J_{\mathrm{sl}}=0,20 \mathrm{~cm} / \mathrm{s} ;[D F 250]=4-62 \mathrm{mg} / \mathrm{L}$.

\section{CONCLUSÕES}

Com o sistema desenvolvido foi possível medir, controlar e monitorar o tamanho de bolha entre 1,0-3,0 mm, assim como medir e monitorar os valores de holdup e Sb (bubble surface área flux).

A estabilização do tamanho de bolha na coluna de flotação ocorreu a partir de 20 a 30 minutos de operação e foi dependente da dosagem de espumante empregada, conforme sugerido pelas medidas de holdup.

O sistema representa um grande avanço para a realização de estudos científicos sobre a flotação, pois servirão para aumento do controle das condições operacionais dos ensaios de flotação, assim como maior entendimento e conhecimento do efeito da distribuição do tamanho de bolha no processo de flotação para diferentes minérios. 


\section{REFERÊNCIAS BIBLIOGRÁFICAS}

1. CHEN, F., GOMEZ, C.O., FINCH, J.A. Bubble size measurement in flotation machines. Minerals Engineering, v.14, n.4, p.427-432, 2001.

2. CHO, Y.S., LASKOWSKI, J.S. Effect of flotation frothers on bubble size and foam stability. International Journal of Mineral Processing, v.64, p.69-80, 2002.

3. FINCH, J. A., DOBBY, G. S., "Column Flotation", Pergamon Press, 1990, p. 180.

4. FINCH, J., XIAO, J., HARDIE, C., GOMEZ, C.O. Gas dispersion properties: Bubble surface area flux and gas holdup. Minerals Engineering, v.13, n.4, p.365-372, 2000.

5. GORAIN, B.K., FRANZIDIS, J.-P. MANLAPIG, E.V. Studies on impeller type, impeller speed and air flow rate in industrial scale flotation cell - part 1: Effect on bubble size distribution. Minerals Engineering, v.8, n.6, p.615-635, 1995.

6. GORAIN, B.K., FRANZIDIS, J.-P. MANLAPIG, E.V., Studies on impeller type, impeller speed and air flow rate in industrial scale flotation cell - part 4: Effect of bubble surface area flux on flotation performance. Minerals Engineering, v.10, n.4, p.639-654, 1997.

7. GRAU, R.A., HEISKANEN, K. Visual technique for measuring bubble size in flotation machines. Minerals Engineering, v.15, p.507-513, 2002.

8. HERNANDEZ-AGUILAR, J.R., COLEMAN, R.G., GOMEZ, C.O., FINCH, J.A. A comparison between capillary and imaging techniques for sizing bubbles in flotation systems. Minerals Engineering, v.17, n.1, p.53-61, 2004.

9. OLIVEIRA, C., SEGER, M.A., RODRIGUES, R.T. Desenvolvimento de um equipamento para avaliação da dispersão de bolhas em células de flotação. In: Encontro Nacional de Tratamento de Minérios e Metalurgia Extrativa. Anais do XXIV Encontro Nacional de Tratamento de Minérios e Metalurgia Extrativa; 2011; Salvador, Brasil. p. 115-124.

10. POMPEO, A.A., OLIVEIRA, C., SEGER, M.A., LOTTERMANN, M.R., RODRIGUES, R.T. Avaliação do tamanho de bolha produzido por um sparger cominco. In: Encontro Nacional de Tratamento de Minérios e Metalurgia Extrativa. Anais do XXV Encontro Nacional de Tratamento de Minérios e Metalurgia Extrativa; 2013; out. 20-24: Goiânia, Brasil. p. 61-69.

11. RODRIGUES, R.T. Desenvolvimento da Técnica LTM-BSizer para a Caracterização de Bolhas e Avaliação de Parâmetros no Processo de Flotação. [Tese de Doutorado]. Programa de PósGraduação em Engenharia de Minas, Metalúrgica e de Materiais - PPGEM/UFRGS; 2004. 